\title{
Analysis of Regulatory T Cell Subsets and Their Expression of Helios and PD-1 in Patients with Hashimoto Thyroiditis
}

\author{
Yifang Hu $\mathbb{D}^{\mathbb{D}}$, Lijuan Zhang, Huanhuan Chen, Xiaoyun Liu $\mathbb{D}$, Xuqin Zheng, He Shi, \\ Lin Jiang, and Dai Cui $\mathbb{D}$ \\ Department of Endocrinology, Nanjing Medical University First Affiliated Hospital, Nanjing, China \\ Correspondence should be addressed to Dai Cui; cui_dai@163.com
}

Received 5 November 2018; Revised 26 February 2019; Accepted 16 April 2019; Published 13 May 2019

Academic Editor: Mario Rotondi

Copyright (c) 2019 Yifang Hu et al. This is an open access article distributed under the Creative Commons Attribution License, which permits unrestricted use, distribution, and reproduction in any medium, provided the original work is properly cited.

\begin{abstract}
Hashimoto thyroiditis (HT) is an autoimmune disease that presumably arises consequent to loss of immune tolerance to autoantigen in thyroid. Regulatory T cells (Tregs) are considered to play a vital role in maintaining the immune balance, as they own intensive suppressive function. This study was undertaken to analyze numbers of Tregs and their expressions of Helios and PD-1 in HT patients. It also aimed to explore the relationship of these with thyroid function and specific autoantibodies. Peripheral blood mononuclear cells (PBMCs) were extracted from blood of 20 healthy controls (HC) and $42 \mathrm{HT}$ patients with varying thyroid functions (10 overt hypothyroidism, 12 subclinical hypothyroidism, and 20 euthyroidism). We performed flow cytometry analysis in PBMCs to detect $\mathrm{CD} 4^{+} \mathrm{CD} 25^{+}$Foxp $3^{+}$Tregs and their subsets, including CD45RO $\mathrm{F}^{+}$Foxp $3{ }^{\text {high }}$ activated Treg cells (aTregs), $\mathrm{CD}_{45 \mathrm{RO}^{-}}$Foxp ${ }^{\text {low }}$ resting Tregs cells (rTregs), and CD45RO ${ }^{+}$Foxp $3^{\text {low }}$ secreting Treg cells (sTregs), as well as the expression of Helios and PD-1 on these cells. The results showed that the percentage of Tregs, aTregs was significantly lower in HT patients and it showed inverse correlation to thyroid function states, in comparison with these in healthy controls. In addition, patients with HT showed decreased expression of Helios in aTregs, while having increased expression of PD-1 in Tregs and sTregs. The levels of Tregs, aTregs, and Helios expressing aTregs were all negatively correlated with antithyroid antibodies. In conclusion, the deficiency of Tregs frequency and aberrant expressions of Helios and PD-1 may possibly contribute to thyroid immune damage in HT.
\end{abstract}

\section{Introduction}

Hashimoto thyroiditis (HT), the most common autoimmune thyroid disease (AITD), is an organ-specific immune disease characterized by immune $\mathrm{T}$ cells and antibody-mediated process [1]. It is suggested that genetic and environmental factors result in the loss of self-tolerance to thyroid antigens and $\mathrm{CD}^{+} \mathrm{T}$ cell activation, which triggers overflowing immune responses, and eventually induce lymphocytic infiltration within thyroid and increase serum antithyroperoxidase antibody (TPOAb) and antithyroglobulin antibody (TGAb) [2] . Meanwhile, there is a crowd of cells with immunosuppressive function. Among these, regulatory $\mathrm{T}$ cells (Tregs) play a crucial role in maintaining homeostasis by dampening and preventing the excessive inflammatory reactions. Previous studies have exposed the evidence that the developmental or functional anomalies of Tregs are tightly correlated with Hashimoto thyroiditis [3-5].
In recent years, advances in our cognition of Tregs have resulted in the discovery of various subtypes, including mainly IL-10-producing Trl (type 1 Treg), TGF- $\beta$-producing $\mathrm{CD}^{+}$Th3 (T helper 3), and $\mathrm{CD} 4^{+} \mathrm{CD} 25^{+}$Foxp3 Tregs. Among these, $\mathrm{CD} 4^{+} \mathrm{CD} 25^{+} \mathrm{Foxp}^{+}{ }^{+} \mathrm{T}$ cells have been a typical and recognized characterization of Tregs up to now. Foxp3 is a master transcription molecule and functional regulator of Tregs, which plays a crucial role in conferring suppressive function to $\mathrm{CD} 4^{+} \mathrm{CD} 25^{+}$Tregs by amplifying the expression of Treg cell-type gene such as CD25, IL-10, and CTLA-4 [6]. In addition, there is a couple of specific surface marker CD45RO/CD45RA, which is coded by a single gene on chromosome 1 (1q32), contributing to the development and progression of $\mathrm{CD}^{+}$lymphocytes. According to the division of different phenotypes, $\mathrm{T}$ lymphocytes are classified into naive $\mathrm{T}$ cell (CD45RA+ or CD45RO- $\mathrm{T}$ cells) and induced $\mathrm{T}$ cells (CD45RA- or CD45RO+ T cells), which are formed in peripheral blood by cytokines stimulation [7]. Recent 
studies $[8,9]$ have shown that the combinatorial expression of Foxp 3 and CD45RO can separate $\mathrm{CD} 4^{+} \mathrm{CD} 25^{+}$Tregs into three functionally and phenotypically distinct subpopulations, consisting of CD45RO ${ }^{+}$Foxp $3^{\text {high }}$ activated regulatory $\mathrm{T}$ cells (aTregs), CD45RO ${ }^{-}$Foxp $3{ }^{\text {low }}$ resting regulatory $\mathrm{T}$ cells (rTregs), and CD45RO ${ }^{+}$Foxp $3{ }^{\text {low }}$ cytokine-secreting regulatory T cells (sTregs). The former two subpopulations confer suppressive function, and aTregs possess highly suppressive ability in vivo. However, sTregs are nonsuppressive and can secrete large amounts of cytokines, such as IL-17, IL-2, and IFN- $\gamma[10,11]$. Meanwhile, relying on CD45RO expression, $\mathrm{CD} 4^{+} \mathrm{CD} 25^{-}$effector $\mathrm{T}$ cells can be divided into $\mathrm{CD} 45 \mathrm{RO}^{-}$ naive $\mathrm{T}_{\text {effect }}$ and $\mathrm{CD} 45 \mathrm{RO}^{+}$memory $\mathrm{T}_{\text {effect }}$ cells. The later one could respond to antigen and display immediate effector function.

Although almost all animal experiments [3, 12-14] have revealed that the absence of Tregs may result in spontaneous progress of autoimmune thyroiditis, the investigation of Tregs in $\mathrm{HT}$ patients is not as unambiguous. Some clinical evidence $[15,16]$ has supported the decreased frequency of circulating Tregs in human subjects with HT, but several conflicting findings were also reported. For example, Bossowski et al. [5] observed lower proportions of $\mathrm{CD} 4^{+} \mathrm{CD} 25^{\text {high }}$ and $\mathrm{CD}^{+}{ }^{+} \mathrm{FoxP}^{+}$, but there were no significant reductions of $\mathrm{CD} 4^{+} \mathrm{CD} 25^{+} \mathrm{CD} 127^{\text {low }}$ Foxp $^{+}$and $\mathrm{CD} 4^{+} \mathrm{CD} 25^{\text {int }}$ Tregs in children with AITD. Marazuela et al. [17] suggested that the levels of $\mathrm{CD}_{6} 9^{+} \mathrm{NKG} 2 \mathrm{D}^{+} \mathrm{IL}-10^{+}$Tregs were increased in peripheral blood from AITD adults. With regard to these results, the different detection markers or subsets with distinct phenotypes may attribute to such discrepancy. Therefore, we mainly focus on investigating the most physiologically relevant $\mathrm{CD} 4{ }^{+} \mathrm{CD} 25^{+}$Foxp $^{+}$Tregs in patients with newly diagnosed HT. Given the dissection of Tregs into subpopulations and little data reported in autoimmune thyroiditis, we sought to identify the proportional change of Tregs subpopulations in normal and HT states.

Beyond estimating Tregs number, research on functional analysis of Tregs in AITD is limited and inconsistent. It has been indicated that IL-10, IL-2, IL-35, and TGF- $\beta$ could be critical cytokines for Tregs activities [18-20]. Of note, two recent markers Helios and $\mathrm{PD}-1$ may also exert vital function in regulating Treg cell peripheral tolerance and autoimmunity. Helios, a member of the Ikaros family, is regarded as important mediator for Tregs since it can upregulate Foxp3 expression by attaching to the Foxp3 promoter [21, 22]. It has been acknowledged that Tregs coexpressing Foxp3 and Helios possess better suppressive abilities than $\mathrm{CD} 4^{+} \mathrm{CD} 25^{+}$ Tregs [23]. To the best of our knowledge, there has not been any research on Foxp $3^{+}$Helios $^{+}$Tregs in the field of thyroid reported. $\mathrm{PD}-1$ belongs to $\mathrm{B} 7$ family and delivers inhibitory signal to prevent immune damage when binding to its ligands PD-L1. In human, the role of PD-1/PD-L1 pathway has been observed in various autoimmune diseases such as rheumatoid arthritis (RA), systemic lupus erythematosus (SLE), multiple sclerosis (MS), and Type 1 diabetes mellitus (T1DM) [24], while little is known in autoimmune thyroiditis. Given that, studies exploring the possible associations of PD-1 and Helios expressing Tregs with HT are warranted.
In this study, we attempted to perform a quantitative and functional analysis of $\mathrm{CD} 4{ }^{+} \mathrm{CD} 25^{+}$Tregs and its diverse subsets in $\mathrm{HT}$ patients due to inconsistent results and limited reports. Considering that HT would suffer different extent immune damage based on frequency of antibodies and then experience three clinical stages defined by thyroid function (euthyroidism, subclinical hypothyroidism, and overt hypothyroidism), Tregs changes and correlations with thyroid autoantibodies are necessary to be recognized in depth as well.

\section{Materials and Methods}

2.1. Subjects. HT patients were recruited from the Endocrinology Outpatient Department of the First Affiliated Hospital of Nanjing Medical University. A total of 42 patients with HT were enrolled in the study according to the following criteria: (1) newly diagnosed HT patients who had never been treated with thyroid hormone replacement or any immune regulators. (2) Laboratory examination showed a normal or increased thyroid-stimulating hormone (TSH) level with elevated antibodies, TPOAb $(>34 \mathrm{IU} / \mathrm{mL})$ or/and TGAb (> $115 \mathrm{IU} / \mathrm{mL})$. (3) Ultrasonography (US) demonstrated a diffuse, enlarged, and heterogeneous thyroid. Patients were classified into three subgroups on the basis of thyroid hormone levels, comprising euthyroidism (eHT), subclinical hypothyroidism (sHT), and overt hypothyroidism (oHT). The laboratory diagnosis of eHT was HT patients with normal levels of FT3, FT4, and TSH; sHT group was HT patients with elevation in levels TSH along with normal levels of FT3 and FT4; oHT group was HT patients with deficient levels of FT4 and raised levels of TSH. During the same period, age- and sex-matched 20 healthy euthyroid volunteers with negative antithyroid antibodies and no history of AITD were recruited as healthy controls (HC).

Exclusion criteria for all subjects were as follows: (1) presence of other autoimmune diseases, such as SLE, RA, MS, or T1DM, (2) acute infections or septicemia, (3) severe systemic disease or chronic disease, (4) current pregnancy and lactation, and (5) the use of anti-inflammatories or immunomodulators.

The study was approved by the Ethics Committee of Nanjing Medical University First Affiliated Hospital (2017SR-141). Informed consents were obtained from all subjects included in the study.

2.2. Blood Samples. Peripheral venous blood $(3 \mathrm{ml})$ was drawn from the enrolled subjects after signing the informed consent. Fresh blood samples collected in vacutainer tubes (green head) containing $0.2 \mathrm{ml}$ heparin were prepared for peripheral blood mononuclear cells (PBMCs).

\subsection{Flow Cytometric Analysis}

(1) Sample Preparation. PBMCs were aseptically isolated using Ficoll-Hypaque density centrifugation within 2 hours of blood being drawn. In brief, blood samples were diluted 1:1 with RPMI-1640 (Gibco, Grand Island, USA) and 
TABLE 1: Clinical and demographic features of the participants.

\begin{tabular}{lccccc}
\hline & HC & eHT & sHT & oHT & HT \\
\hline Number & 20 & 20 & 12 & 10 & 42 \\
Sex (F/M) & $15 / 5$ & $19 / 1$ & $9 / 3$ & $9 / 1$ & $37 / 5$ \\
Age (years) & $38.85 \pm 8.07$ & $41.4 \pm 13.55$ & $40.9 \pm 11.89$ & $37.2 \pm 14.59$ & $40.29 \pm 13.15$ \\
FT3 (pmol/L) & $4.48(4.28-5.01)$ & $4.35(3.94-4.68)$ & $4.42(4.18-5.1)$ & $3.34(2.52-4.26)^{\mathrm{a}}$ & $4.21(3.7-4.72)$ \\
FT4 (pmol/L) & 15.5 & 15.28 & 14.92 & $9.67^{\mathrm{abc}}$ & 14.46 \\
TSH (mIU/L) & $(14.27-16.65)$ & $(13.97-18.01)$ & $(13.5-16.35)$ & $(5.11-10.86)$ & $(12.15-16.54)$ \\
TPOAb (IU/ml) & $2.25(1.82-2.84)$ & $2.11(1.59-3.42)$ & $5.82(4.68-6.7)^{\mathrm{ab}}$ & $36.9(23.68->100)^{\mathrm{ab}}$ & $4.45(2.11-11.02)$ \\
& 13.0 & $173.2^{\mathrm{a}}$ & $248.45^{\mathrm{a}}$ & $473.5^{\mathrm{ab}}$ & 238.55 \\
TgAb (IU/ml) & $(6.5-22.75)$ & $(69.97-235.77)$ & $(172.88-562.6)$ & $(306.25->600)$ & $(160.9-479.45)$ \\
& 32.21 & $534.35^{\mathrm{a}}$ & $402.05^{\mathrm{a}}$ & $1053.3^{\mathrm{a}}$ & 545.75 \\
$\mathrm{D}$ & $(20.25-51.08)$ & $(310.15-623.48)$ & $(146.78-549.7)$ & $(797.1-1835)$ & $(345.78-886.7)$ \\
\hline
\end{tabular}

Data was expressed as mean \pm SD or median (25th-75th percentile) on the basis of the distribution. HC: healthy controls; HT: Hashimoto thyroiditis; $e H T$ : euthyroidism; $s H T$ : subclinical hypothyroidism; $o H T$ : clinical hypothyroidism; F: female; $M$ : male; FT3: free triiodothyronine; FT4: free thyroxine; TSH: thyroid stimulating hormone; $T P O A b$ : antithyroid peroxidase antibodies; $\operatorname{Tg} A b$ : antithyroglobulin antibodies.

${ }^{a} P<0.05$, comparison with health controls.

${ }^{\mathrm{b}} P<0.05$, comparison with eHT group.

${ }^{\mathrm{c}} P<0.05$, comparison with sHT group.

slowly dropped in the inclined tubes with lymphoprep, then centrifuged for $30 \mathrm{~min}$ at $800 \mathrm{~g}, 20^{\circ} \mathrm{C}$. Mononuclear cells acquired from the middle gradient interface were washed twice and suspended in phosphate buffered saline (PBS).

(2) Cell Surface Staining. Nearly $1 \times 10^{6}$ PBMCs were stained and incubated for $30 \mathrm{~min}$ at $20^{\circ} \mathrm{C}$ in darkness with $3 \mu \mathrm{l}$ (according to optimisation assays) of following monoclonal antibodies: anti-CD3 APC-H7 (BD Bioscience, New Jersey, USA), anti-CD4 BV510 (BD Bioscience, New Jersey, USA), anti-CD25 PE-cy7 (BD Bioscience, New Jersey, USA), antiCD45RO BV421 (BD Bioscience, New Jersey, USA), and anti-PD-1 PE (BioLegend, Santiago, USA). Then, cells were washed twice in $2 \mathrm{ml} \mathrm{PBS}$ at $3000 \mathrm{r} 4^{\circ} \mathrm{C}$ for $5 \mathrm{~min}$ and remove the supernatants.

(3) Intracellular Cytokine Staining. After fixation and permeabilization with Fix/Perm kit (eBioscience, San Diego, CA, USA) at $4^{\circ} \mathrm{C}$ for $45 \mathrm{~min}$, cells were stained intracellularly with $4 \mu \mathrm{l}$ of anti-Foxp3 Alexa Fluor 647 and anti-Helios FITC (BioLegend, Santiago, USA) at $20^{\circ} \mathrm{C}$ for $30 \mathrm{~min}$ in darkness. Then, cells were washed twice in $2 \mathrm{ml}$ perm/wash buffer $(1 \times)$ and centrifuged $5 \mathrm{~min}$ at $3000 \mathrm{r} 4^{\circ} \mathrm{C}$. All the steps of the experiment were strictly performed according to the manufacturer's instructions.

(4) Multicolor Flow Cytometric Analysis. The stained cells were identified, quantified, and classified on seven-color flow cytometry (FCM, FACS Verse, BD Biosciences, USA) and Flow Jo 10. Lymphocytes were first gated, followed by CD4+ Th cells based on CD3 and CD4 expression. Quadrants of Tregs were set according to the expression of CD25 and Foxp3 on CD4+ cells. The CD4+ cells without CD25 and Foxp3 expression were defined as Teffect cells. The three Treg subsets were differentiated by the levels of CD45RO and Foxp3, including Foxp $3{ }^{\text {hi }} \mathrm{CD}_{45 \mathrm{RO}^{+}}$aTregs,
CD45RO ${ }^{+}$Foxp $^{\text {lo }}$ sTregs, and $\mathrm{CD}^{2} 5 \mathrm{RO}^{-} \mathrm{Foxp}^{\text {lo }}$ rTregs. Similarly, Teffect cells could be separated into memory $\mathrm{CD}_{45 \mathrm{RO}^{+}}{ }^{+}$Teffect and initial CD45RO ${ }^{-}$Teffect cells. Based upon above phenotypes, we further analyzed Helios ${ }^{+}$and PD-1 levels in Treg, Teffect cells and their subsets.

2.4. Statistical Analysis. All statistical data were calculated and analyzed by SPSS 25 (IBM SPSS Software, Inc., Chicago, USA) and GraphPad Prism 5.0 software (GraphPad Software, Inc., San Diego, CA, USA). Continuous data were represented as mean \pm standard deviation (SD) or median with percentiles (25-75 percentiles) on the basis of the distribution, which was detected by Shapiro-Wilk normality test. For normally distributed data, comparisons between two groups were analyzed by independent-sample $\mathrm{T}$ test, while more groups by one-way ANOVA and multiple comparison test (LSD, Tukey). For abnormal distribution series, we used the nonparametric tests to examine the differences within groups or between groups. Specifically, the Kruskal-Wallis test was used in comparisons among three or more groups, Wilcoxon-Mann-Whitney test was utilized to test the heterogeneity between two groups, and unpaired/Welch's correction was applied for samples with unequal variances. Pairwise correlations between different markers were performed by Spearman's correlation test. $P$ values $<0.05$ were considered to be statistically significant.

\section{Results}

3.1. Characteristics of the Subjects. Clinical and demographic features of patients with HT and HC were shown in Table 1. Samples derived from $20 \mathrm{HC}, 20 \mathrm{eHT}, 12 \mathrm{sHT}$, and $10 \mathrm{oHT}$ were included in our study. There was a similar age distribution among all groups. Most of the participants were females, which was consistent with the epidemiological characteristic of HT. When compared to HC group, the FT3 and FT4 levels 
were significantly lower ( $P=001, P<0.0001$, respectively) in oHT group, while TSH was higher in both $\mathrm{sHT}$ and oHT group $(P<0.0001, P=0.002$, respectively). A statistically significant increase in TPOAb and TgAb was observed in all subgroups of HT patients, but having a marked increase in overt hypothyroidism $(P<0.0001)$.

3.2. The Percentage of Treg Cells. Compared to HC, the analysis of Treg cells in patients with HT revealed a statistically significant decrease in the percentage of Treg cells (4.19 \pm $0.93 \%$ vs. $5.92 \pm 1.27 \%, P<0.0001$, Figure $2(\mathrm{a})$ ), but no difference in Teffect cells. To further explore the differences, we performed a multiple analysis of different subsets in the stage of HT progress. The aTregs in HT and all subgroups were significantly lower than in $\mathrm{HC}$ (HT vs. HC, $8.5 \pm 3.35 \%$ vs.12.75 $\pm 4.52 \%, P<0.0001$, Figure $2(\mathrm{~b}))$, while neither rTregs $(P>0.05$, Figure $2(\mathrm{c}))$ nor sTregs $(P>0.05$, Figure $2(\mathrm{~d}))$ were different between groups. In HT patients, Treg cells, as well as aTregs, seemed to trend downward between eHT, sHT, and oHT subgroups; there was a significant difference between eHT and oHT patients. Additional analysis of Teffect subgroups in HT patients showed that the proportion of $\mathrm{CD}_{45 \mathrm{RO}^{+}}$Teffect in CD $4^{+} \mathrm{T}$ cells was significantly enhanced $(46.65 \pm 11.11$ vs. $35.25 \pm 11.47, P<0.0001$, Figure $2(\mathrm{e}))$, but conversely $\mathrm{CD} 45 \mathrm{RO}^{-}$Teffect was reduced compared to $\mathrm{HC}$ $(46.96 \pm 11.43$ vs. $58.98 \pm 11.58, P<0.0001$, Figure 2 (f)). The representative flow cytometric dot plots and analysis results are shown in Figures 1 and 2.

3.3. Helios Expression in Treg Cells. As shown in Figure 3(a), a high level of Helios was expressed in Tregs, followed by a rather higher expression in aTregs in contrast to Teffect cells with diminished Helios expression. We focused on investigating the proportion of Tregs and subsets expression of Helios between HT patients and healthy controls. There was no significant differences of Helios expression in circulating total Tregs among different groups $(P>0.05$, Figure 3(b)). For Tregs subsets, aTregs exhibited profoundly reduced expression of Helios in HT patients $(P=0.019$, Figure 3(b)) as compared with $\mathrm{HC}$, and the difference was more obvious in hypothyroid patients $(P=0.0028$, Figure $3(b))$. Moreover, we observed the expression of Helios had a positive correlation with the percentage of aTregs in HT patients $\left(\mathrm{r}_{\mathrm{s}}=0.505, P=\right.$ 0.0006 , Figure $3(\mathrm{c})$ ). However, sTregs and rTregs expressing Helios showed no significant differences among all groups $(P$ $>0.05$, Figure 3(b)).

3.4. PD-1 Expression in Treg Cells. We analyzed PD-1 expression in Treg and Teffect cells derived from HC and HT patients, as shown in Figure 4. Similar to previous studies, PD-1 was upregulated on aTregs, sTreg, and CD45RO ${ }^{+}$Teffect cells, while being lowly expressed on rTregs and CD45RO ${ }^{-}$ Teffect cells in both groups. Importantly, compared to HC, expressions of PD-1 on Tregs, sTregs, and CD45RO ${ }^{+}$Teffect cells were significantly higher in HT patients and subgroups. However, there were no significant differences of PD-1 expression on rTregs, aTregs, or CD45RO ${ }^{-}$Teffect cells between groups.
3.5. Correlation Analysis. To examine whether there was any correlation between the percentage of Tregs, Helios or PD-1 expression and clinical characteristics of HT patients, we performed Spearman's correlation analysis on pooled samples. A series of significant relationships were observed. The percentage of Tregs correlated inversely with serum levels of TSH $(r=-0.388, p=0.01)$, TPOAb $(r=-0.446$, $p=0.003)$, and $\operatorname{TgAb}(\mathrm{r}=-0.565, p<0.0001)$, as did the classic Treg subset, aTregs $(r=-0.385, P=0.01 ; \mathrm{r}=-0.394$, $P=0.009 ; \mathrm{r}=-0.506, P=0.0006$, respectively), as shown in Figure 5(a). Additionally, we found a significant positive correlation between Teffect ratio and TPOAb $(r=0.309$, $P=0.04)$ or $\mathrm{TgAb}(\mathrm{r}=0.476, P=0.0015)$, as shown in Figure 5(b). Moreover, the proportion of aTreg expressing Helios showed a positive correlation with serum FT3 levels ( $\mathrm{r}$ $=0.343, P=0.0263)$, whereas inverse correlation with serum TSH concentrations $(r=-0.374, P=0.015)$, and TPOAb $(r$ $=-0.497, P=0.0008)$ or TgAb titers $(r=-0.435, P=0.004)$, as shown in Figure 5(c). However, there were no significant associations between PD-1 status and clinical parameters in HT, as shown in Figure 5(d).

\section{Discussion}

The pathogenesis of HT so far has not been completely explored, although HT is widely considered to be a Th1mediated autoimmune disease. Various studies have demonstrated that Tregs play an important role in preventing autoimmunity and restraining responses of effector $\mathrm{T}$ cells, but the frequency and function of Tregs in the progression of AITD are discrepant $[4,25,26]$. Defective Tregs frequency has been widely reported to exist in Graves' disease [27-32], while less evidence is available regarding Tregs in HT. In this regard, we assessed the classical $\mathrm{CD} 4^{+} \mathrm{CD} 25^{+}$Foxp $3^{+}$Tregs number and two key functional indicators in $\mathrm{HT}$ patients and HC.

In this study, we found that the percentage of Tregs was apparently reduced in PBMC of HT patients compared to controls. Previously, significant decreases in the number of $\mathrm{CD}^{+}$Foxp $^{+}$Tregs, $\mathrm{CD} 4^{+} \mathrm{CD} 25^{\text {high }}$ Tregs, $\mathrm{CD} 4^{+} \mathrm{CD} 25^{+} \mathrm{CD} 127^{\text {low }}$ Tregs, and $\mathrm{CD} 4^{+} \mathrm{CD} 25^{\text {high }}$ Foxp $3^{+}$ Tregs were subsequently reported in peripheral blood of HT patients $[5,16,33]$. Our result confirmed the observations from these studies, but some conflicting findings were discovered in other studies. Glick et al. reported that no differences existed in the frequency of $\mathrm{CD} 44^{+} \mathrm{CD} 25^{\text {high }}$ Tregs between AITD, HT, GD, and HC [4]. Marazuela et al. detected a significantly higher proportion of $\mathrm{CD} 4^{+}$Foxp $3^{+}$ Tregs in AITD patients [25]. The discrepancy may be attributed to differences in surface markers, gating strategies of FCM or analysis methods. We investigated the classical $\mathrm{CD} 4^{+} \mathrm{CD} 25^{+} \mathrm{Foxp}^{+}$Treg phenotype which was widely used in autoimmune diseases, but also supplied novel evidence of Treg subpopulations in different clinical stages of HT and their associations with clinical features.

Treg cells were delineated as three subpopulations: CD45RO ${ }^{+}$Foxp $3^{\text {high }}$ aTregs, CD45RO ${ }^{-}$Foxp $3^{\text {low }}$ rTregs, and $\mathrm{CD}_{45 \mathrm{RO}^{+}}$Foxp $^{\text {low }}$ sTregs. Among them, aTreg is a critical 


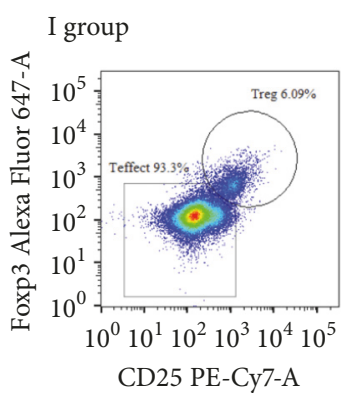

(a)

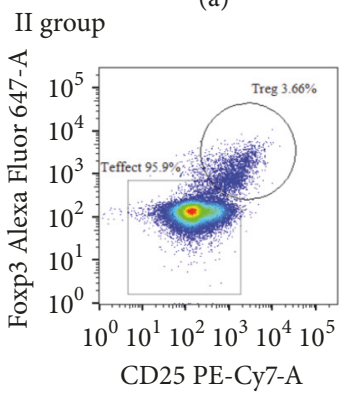

(a)

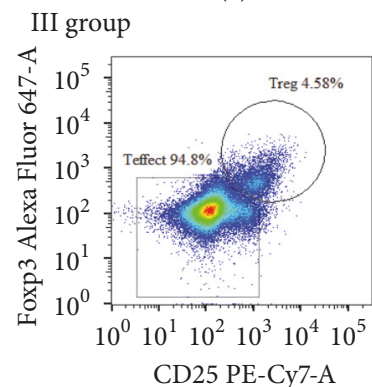

(a)

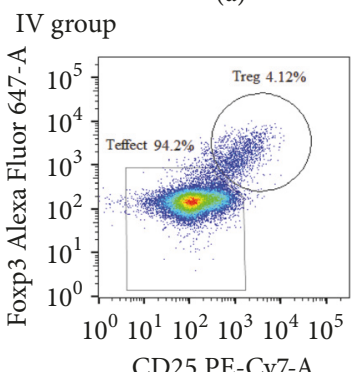

(a)

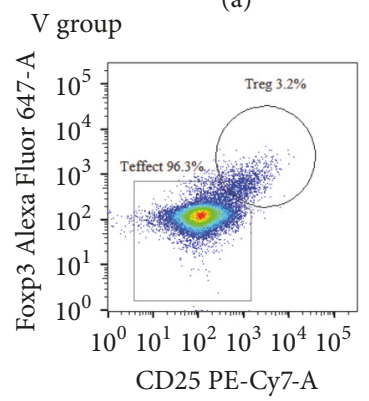

(a)

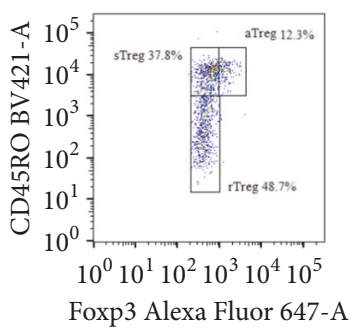

(b)

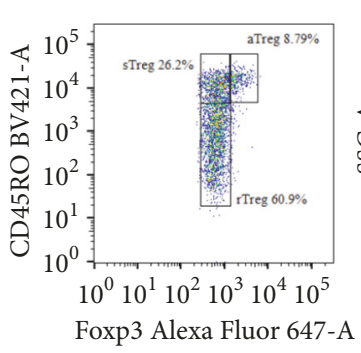

(b)

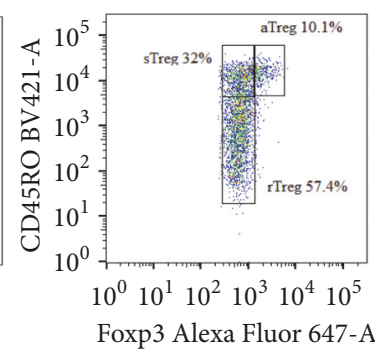

(b)

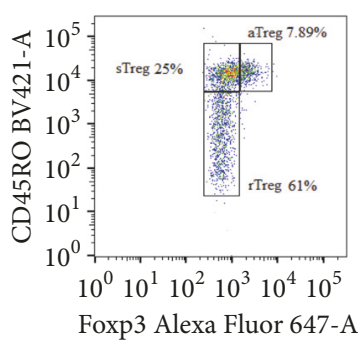

(b)

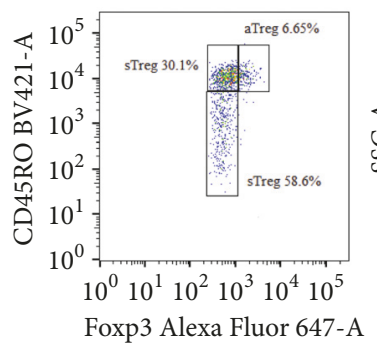

(b)

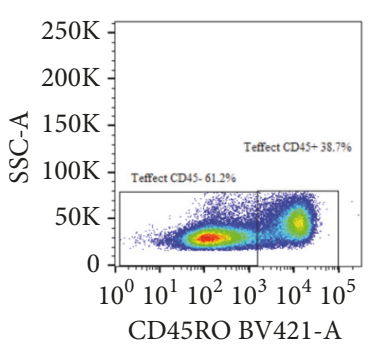

(c)

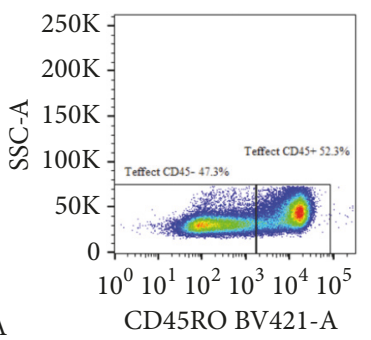

(c)

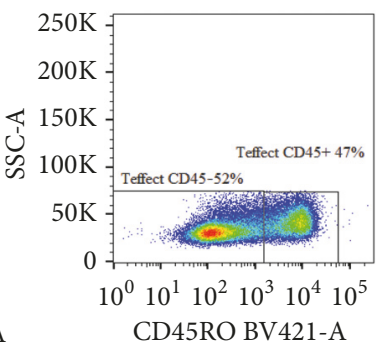

(c)

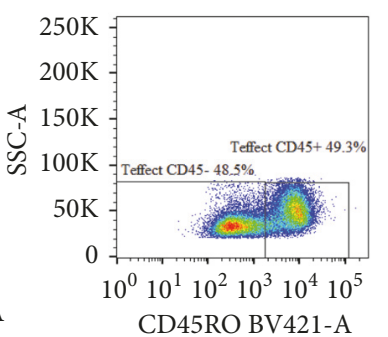

(c)

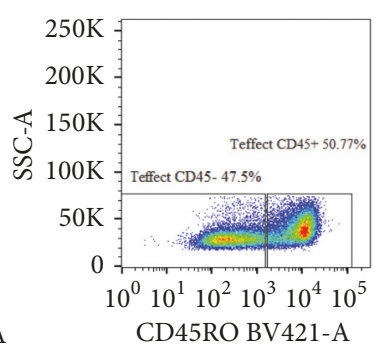

(c)

FIGURE 1: Representative flow cytometric dot plots of the T cell subsets in peripheral blood of subjects. I group represent the healthy controls, II group represent HT patients, III group represent euthyroidism HT, IV group represent subclinical hypothyroidism HT, and V group represent overt hypothyroidism HT. (a) Gating Treg cells (CD25 $5^{+} \mathrm{Foxp}^{+} / \mathrm{CD}^{+} \mathrm{CD} 4^{+} \mathrm{T}$ cells), the remainder was regarded as Teffect cells. (b) Treg cells

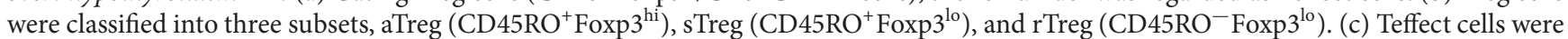
divided into two subsets, CD45RO $\mathrm{Reffect}^{+}$and $\mathrm{CD}^{+} 5 \mathrm{RO}^{--}$Teffect. 


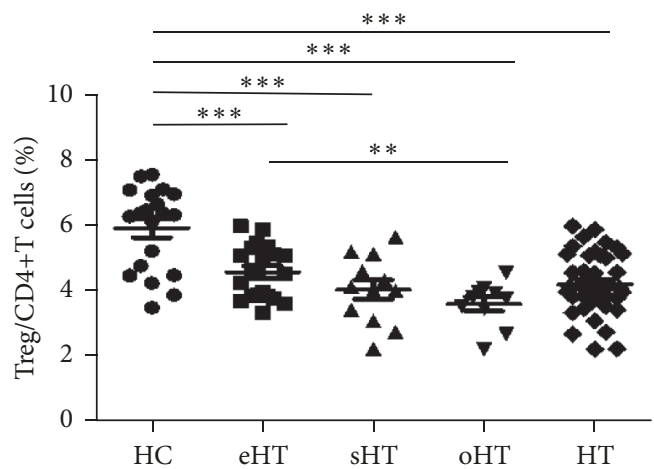

(a)

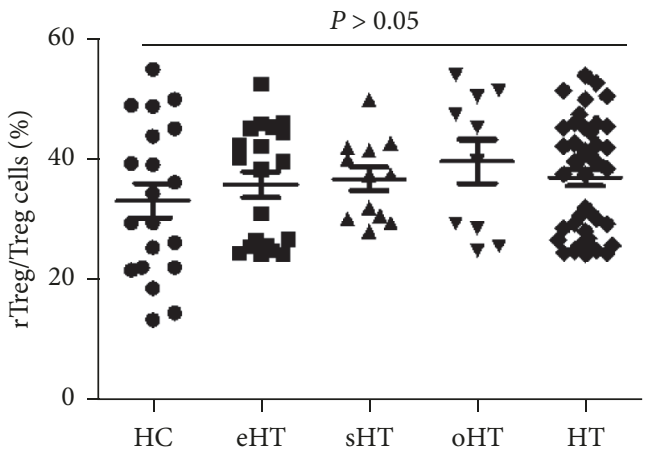

(c)

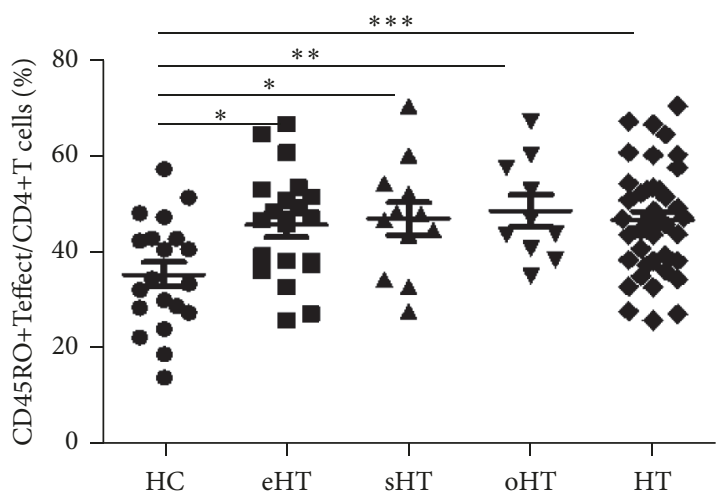

(e)

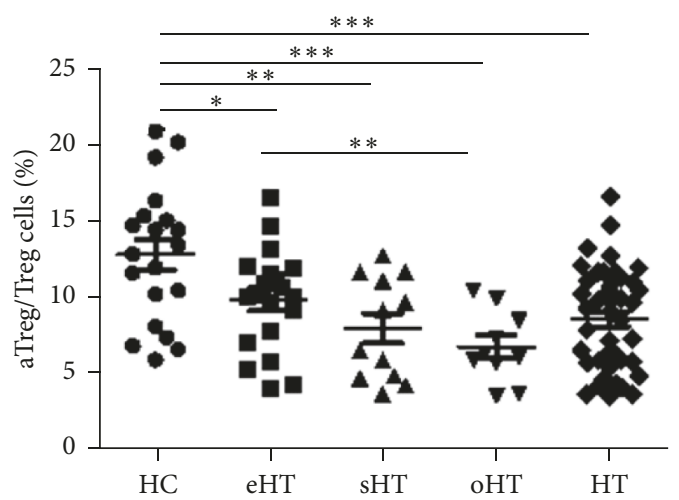

(b)

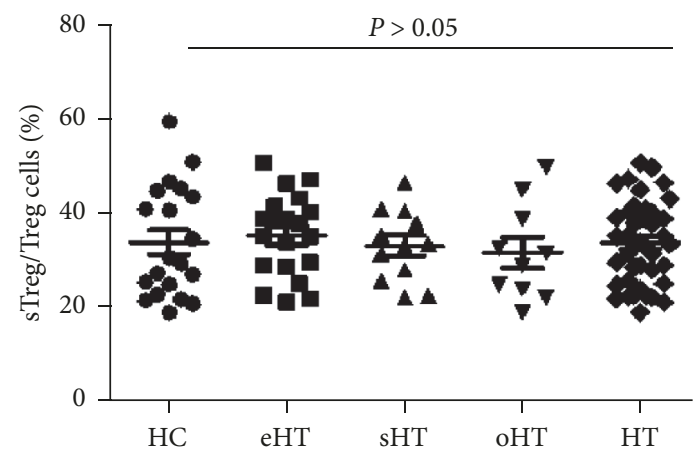

(d)

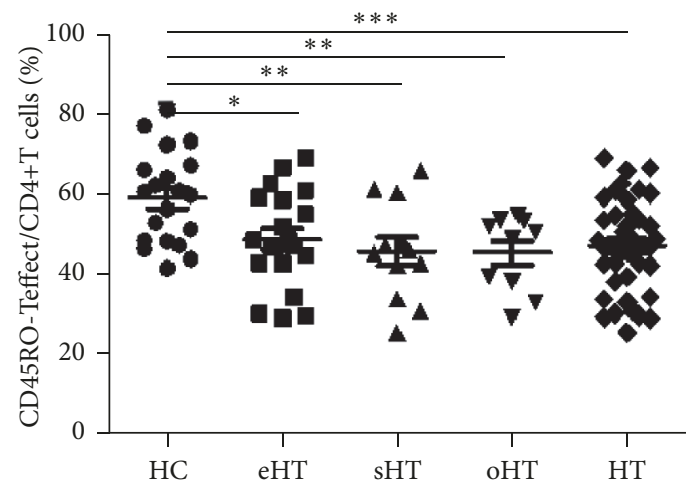

(f)

Figure 2: Differences of T lymphocyte subpopulations in HT patients and healthy controls. Percentages of (a) Treg in CD $4^{+} \mathrm{T}$ cell, (b) aTreg in Treg cells, (c) rTreg in Treg cells, (d) sTreg in Treg cells, (e) CD45RO ${ }^{+}$Teffect in CD $4^{+} \mathrm{T}$ cells, and (f) $\mathrm{CD}_{4} \mathrm{RO}^{--}$Teffect in $\mathrm{CD} 4^{+} \mathrm{T}$ cells $(* \mathrm{P}$ $<0.05, * * \mathrm{P}<0.0075, * * * \mathrm{P}<0.0001)$.

suppressor that inhibits responder cells from proliferating and secreting cytokines. The current study showed that aTregs were significantly decreased in HT patients of the variable disease states accompanying decrement of total Tregs. However, there were no significant changes in the proportion of rTreg or sTreg between HT patients and HC. It has previously been reported that the decrement of aTreg not rTreg contributes to the decline of total Tregs in rheumatoid arthritis (RA) and Behcet's disease (BD) [34]. These facts indicate that the proportional reduction of activated Tregs may participate in the pathogenesis of HT. In addition, we observed that Tregs and aTregs stepwise decreased during the clinical stages of HT and negatively correlated with TSH, TPOAb, or TgAb levels. Presumably, the dynamics of aTregs would appear to reflect the severity and activity of disease. As described in systemic sclerosis (SSc), it showed a gradual decrease of the circulating aTreg subset with the progression of disease [35]. Moreover, Miyara et al. demonstrated that rTregs could be converted into aTregs in vivo, and in active SLE, there was a proportional decrease of aTregs, while having an increase of rTregs [8]. Regarding our study, aTregs decreased gradually along with the increase of TSH, TPOAb, TGAb levels. It was implied that HT disease severity and thyroid dysfunction became more apparent with the decreased 

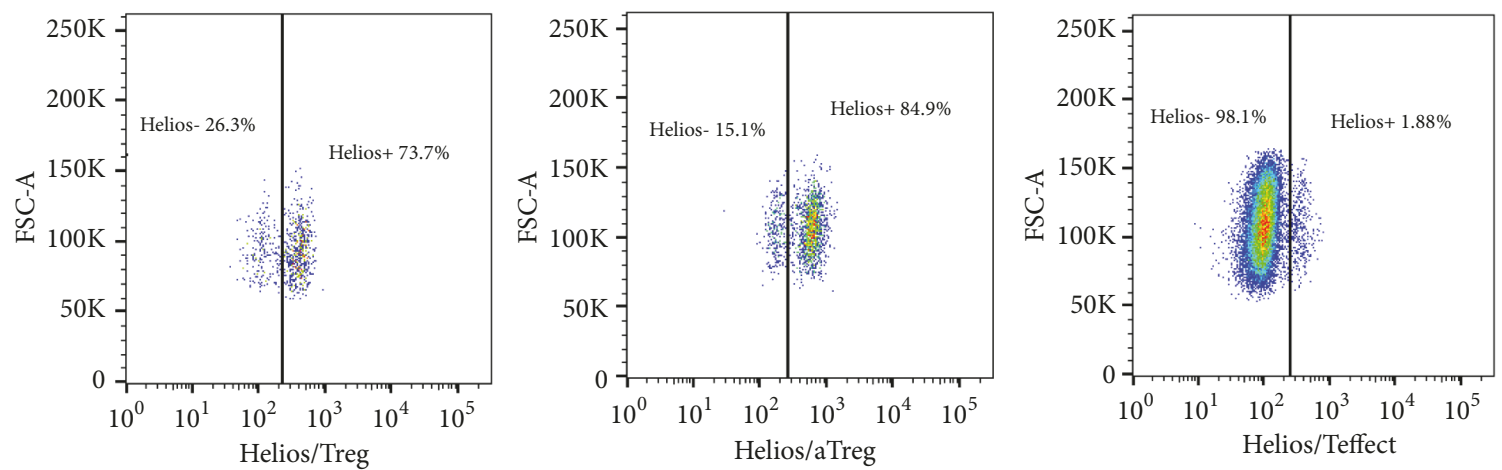

(a)
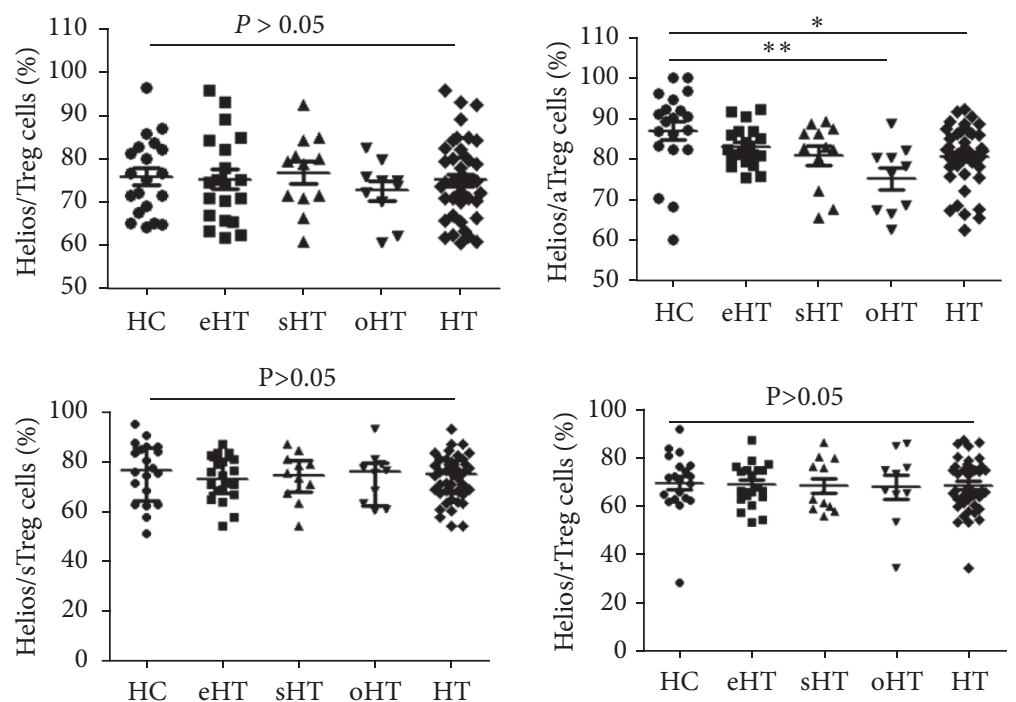

(b)

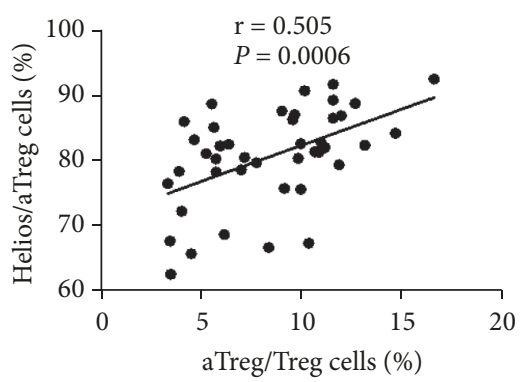

(c)

Figure 3: Helios expression in Treg cells. (a) Representative flow cytometric plots of Helios staining in Treg, aTreg, and Teffect cells from one HT patient. (b) Comparison of Helios expressing Tregs and subsets between different study groups. (c) Correlation between Helios expression and aTreg cells from HT patients $(* \mathrm{P}<0.05 ; * * \mathrm{P}<0.0075 ; * * * \mathrm{P}<0.0001)$.

aTreg cells. Supporting these findings, it was also revealed in HT patients that the proportion of $\mathrm{CD}_{4} 4 \mathrm{RO}^{+}$memory effector $\mathrm{T}$ cells was increased and positively correlated with $\mathrm{TPOAb}$ and $\mathrm{TgAb}$, which was compatible with impairment of immunosuppressive Tregs.

Apart from this, we also assessed the critical function of Tregs in HT patients. Helios is a novel marker and upregulated in Tregs, conferring stable phenotype and stronger immunosuppressive function. It was recently shown that Foxp $3^{+}$Helios ${ }^{+}$Tregs had a greater suppressive ability than either Foxp3 or Helios expressing Tregs, and there was a positive correlation between the two molecules [36]. In our study, aTregs possessed a relatively high expression of Helios, which indicates aTregs may have stronger immunosuppression. However, we found the expression of Helios in aTreg subset was significantly lower in HT patients than in HC. Besides, Helios expressing aTreg was negatively correlated with serum TSH, TPOAb, or TgAb levels. These findings of Helios were in line with those of aTreg in HT patients. It is probably due to the fact that Helios is an intracellular marker, directly attaching to the Foxp3 promoter, thus controlling the suppressive capabilities of Tregs. With respect to the consequence of Helios, we speculate that the reduction of Helios expression preludes the development of HT. However, outcomes of Helios in autoimmune diseases may differ according to the dependency of each disease on Tregs function. Preceding studies reported the increased expression of Helios in RA [22], as well as high levels of Helios ${ }^{+}$Tregs in SLE [37]. In this paper, the role of Helios in HT was studied for the first time, but the exact mechanism remains not well understood. Hereafter, more extensive researches are still needed to explicate in detail the functional importance of Helios in the progression of HT.

PD-1 is considered as another functional biomarker for $\mathrm{CD}^{+} \mathrm{T}$ cells. It is preferentially expressed on activated Treg or Teffect cells. The interaction of PD-1 and PD-L1 can maintain homeostasis between protective responses of Treg and hyperactivated immunoreaction of Teffect cells [38]. The majority of the studies indicated that blocking PD-1/PDL1 pathway masked Treg mediated inhibition of effector 


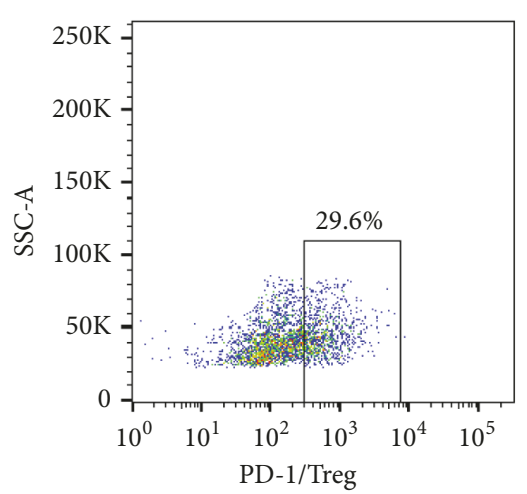

(a)

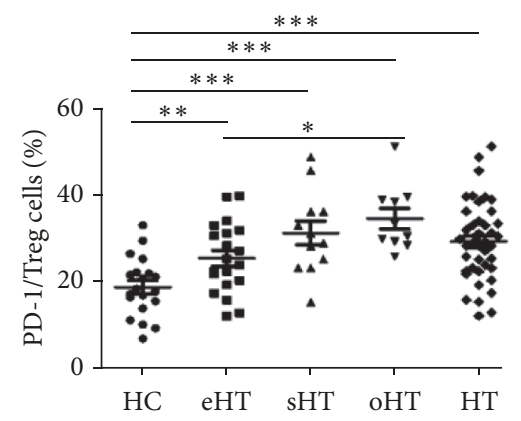

(d)

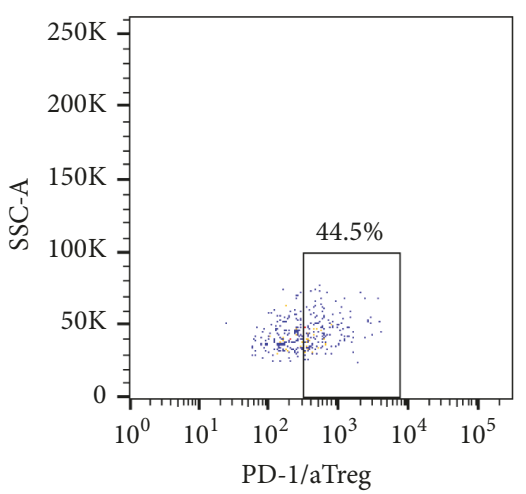

(b)

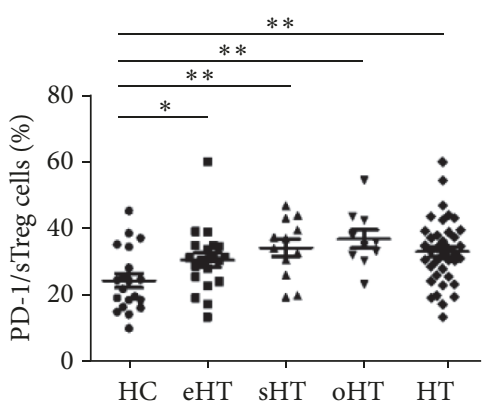

(e)

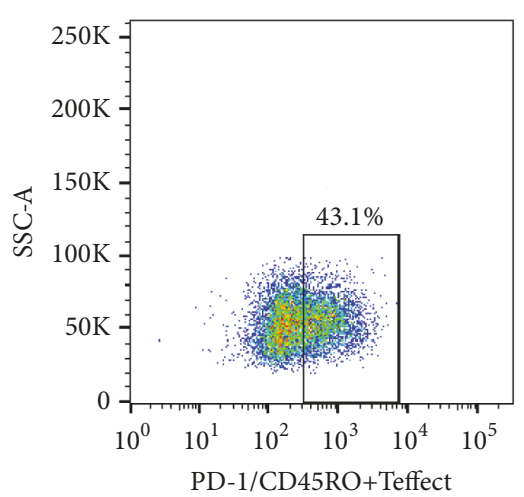

(c)

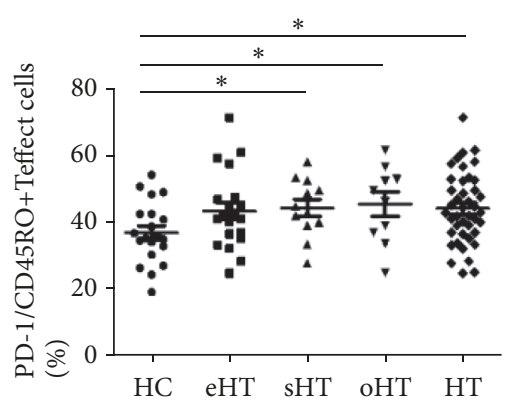

(f)

Figure 4: PD-1 expression on Treg and Teffect cells. Representative flow cytometric plots of PD-1 staining in (a) Treg, (b) aTreg, and (c)

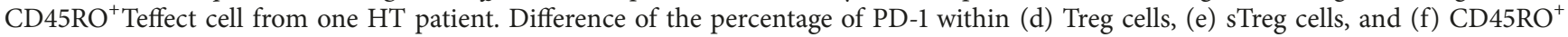
Teffect cells among different groups $(* \mathrm{P}<0.05 ; * * \mathrm{P}<0.0075 ; * * * \mathrm{P}<0.0001)$.

$\mathrm{T}$ cells, thus supporting PD-1-dependent Treg suppressive function [39-42]. Contrary to these findings, Franceschini et al. argued that PD-L1 blockade resulted in expansion of Tregs by restricting STAT- 5 phosphorylation, suggesting PD1/PD-L1 contraregulated Treg function [43]. It was tricky to understand the opposite outcome, perhaps the mechanism of PD-1/PD-L1 pathway was influenced by phenotypic diversity of Tregs or microenvironment of diseases. So far in human autoimmune diseases, there has been reported that the level of PD-1 expression on Tregs is significantly lower in SLE, while higher in active generalized vitiligo (GV) patients compared to the controls $[44,45]$. However, the role of PD-1 has not been studied in thyroiditis. Here, we first proposed that HT patients showed increased PD-1 expression on Treg and Teffect cells, especially on CD45RO ${ }^{+}$Foxp $3^{\text {low }}$ sTreg and $\mathrm{CD}_{45 \mathrm{RO}^{+}}$Teffect subsets. Classically, HT is regarded to be a Th1-mediated disease, caused by strong inflammatory cytokines infiltration. The CD45RO $\mathrm{R}^{+}$Teffect cells are memory effector cells that produce IL-2, IFN- $\gamma$ or IL- 4 and associated with the pathogenesis of HT. Besides, sTregs are considered as nonregulatory $\mathrm{T}$ cells since they have no suppressive properties and emulate Teffect function. Given that PD-1 expresses inhibitory signals, it may be hypothesized that increased PD-1 expression on sTreg and Teffect cells suppressed the proliferation of autoreactive $\mathrm{T}$ cells in a negative feedback manner. However, this hypothesis in HT disease needs further demonstration. Significantly, PD-1 has been emerging as a potential therapeutic target for ameliorating autoimmune disease [46]; the same prospect could be expected in thyroid aspects.

\section{Conclusion}

In this study, our data indicated that HT patients exhibit low percentage of classical Tregs and aTregs, and these cells had dynamic changes in the progression of HT. We also found decreased expression of Helios on aTreg cells in HT, whereas PD-1 expression on sTreg and memory Teffect cells was peripherally expanded. More importantly, Tregs and Helios had various associations with serum levels of thyroid-specific autoantibodies, as well as thyroid function in patients with HT. These findings suggest the change of Tregs number and function might be involved in the immunopathogenesis of HT. However, the relatively small sample size is a limitation to address this issue. More samples and further work is necessary to dissect the exact relationship between Tregs and the pathogenesis of HT. Furthermore, it is speculated that Tregs may serve as a novel therapeutic target, and Tregbased intervention is supposed to be implicated in future therapeutics for HT patients.

\section{Abbreviations}

HT: Hashimoto thyroiditis

HC: Healthy controls 

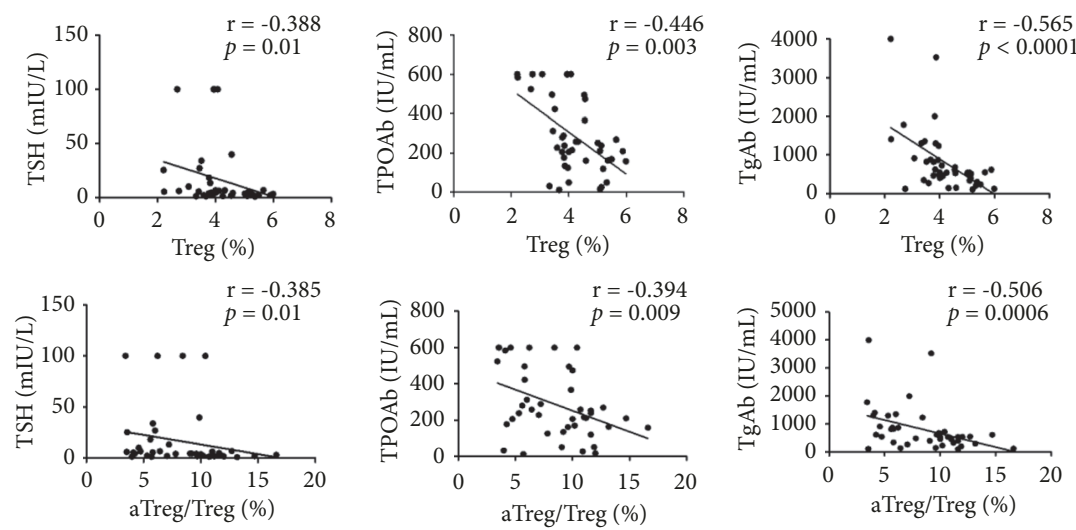

(a)
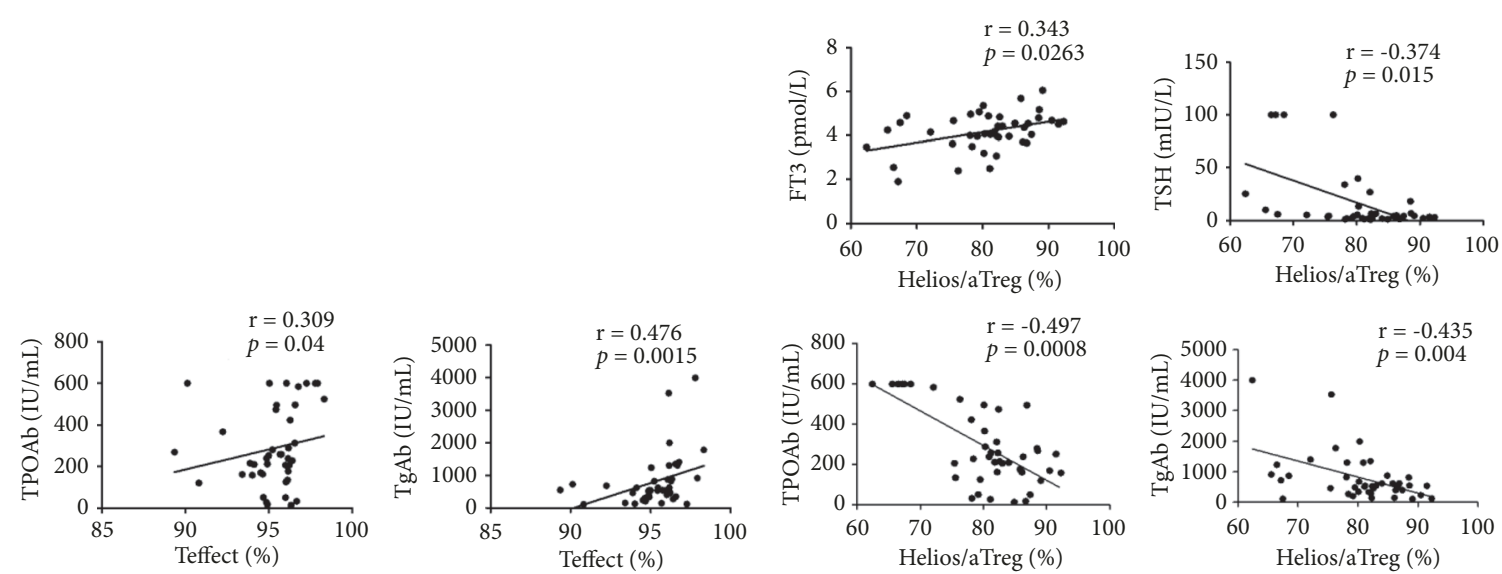

(b)

(c)
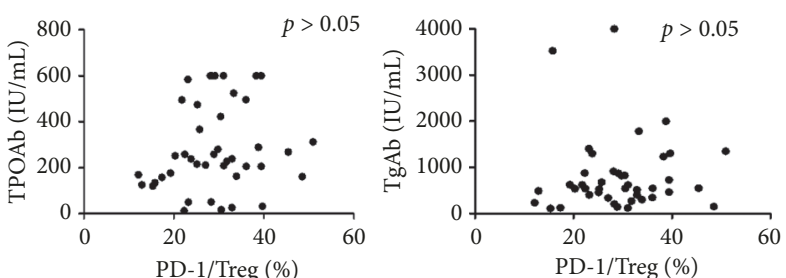

(d)

Figure 5: Spearman pairwise correlation analysis in HT patients between (a) the Treg or aTreg ratio and TSH, TPOAb or TgAb levels. (b) The Teffect ratio and TPOAb or TgAb levels. (c) The proportion of Helios in aTreg and FT3, TSH, TPOAb or TgAb levels. (d) Proportion of PD-1 in Treg and TPOAb or TgAb levels ( $\mathrm{r}$ : Spearman correlation coefficient, significance $\mathrm{P}<0.05$ ).

AITD: Autoimmune thyroid disease

Tregs: Regulatory T cells

aTregs: Activated Treg cells

rTregs: Resting Tregs cells

sTregs: Secreting Treg cells

TPOAb: Antithyroperoxidase antibody

TGAb: Antithyroglobulin antibody

TSH: Thyroid-stimulating hormone

eHT: Euthyroidism

sHT: Subclinical hypothyroidism

oHT: Overt hypothyroidism

PBMCs: Peripheral blood mononuclear cells

PBS: $\quad$ Phosphate buffered saline

FCM: Flow cytometry

RA: Rheumatoid arthritis
SLE: Systemic lupus erythematosus

MS: Multiple sclerosis

T1DM: Type 1 diabetes mellitus

SSc: $\quad$ Systemic sclerosis.

\section{Data Availability}

The data used to support the findings of this study are available from the corresponding author upon request.

\section{Consent}

Informed consents were obtained from all individual participants included in the study. 


\section{Conflicts of Interest}

The authors declare that there are no conflicts of interest in the publication of this paper.

\section{Authors' Contributions}

Yifang $\mathrm{Hu}$ and Lijuan Zhang contributed equally to this work.

\section{Acknowledgments}

This work was supported by the Project of the peak of Six Personnel in Jiangsu Province (2016-WSN-023), the National Natural Science Foundation of China (81670715), and the Priority Academic Program Development of Jiangsu Higher Education Institutions (PAPD).

\section{References}

[1] G. Cogni and L. Chiovato, "An overview of the pathogenesis of thyroid autoimmunity," Hormones, vol. 12, no. 1, pp. 19-29, 2013.

[2] A. Hasham and Y. Tomer, "Genetic and epigenetic mechanisms in thyroid autoimmunity," Immunologic Research, vol. 54, no. 13, pp. 204-213, 2012.

[3] W.-Z. Wei, J. B. Jacob, J. F. Zielinski et al., "Concurrent induction of antitumor immunity and autoimmune thyroiditis in CD4+CD25+ regulatory T cell-depleted mice," Cancer Research, vol. 65, no. 18, pp. 8471-8478, 2005.

[4] A. B. Glick, A. Wodzinski, P. Fu, A. D. Levine, and D. N. Wald, "Impairment of regulatory T-Cell function in autoimmune thyroid disease," Thyroid, vol. 23, no. 7, pp. 871-878, 2013.

[5] A. Bossowski, M. Moniuszko, M. Dabrowska et al., "Lower proportions of $\mathrm{CD} 4+\mathrm{CD} 25^{\text {high }}$ and $\mathrm{CD} 4+$ FoxP3, but not $\mathrm{CD} 4+\mathrm{CD} 25+\mathrm{CD} 127^{\text {low }} \mathrm{FoxP}^{+} \mathrm{T}$ cell levels in children with autoimmune thyroid diseases," Autoimmunity, vol. 46, no. 3, pp. 222-230, 2013.

[6] R. M. Samstein, A. Arvey, S. Z. Josefowicz et al., "Foxp3 exploits a pre-existent enhancer landscape for regulatory $\mathrm{T}$ cell lineage specification," Cell, vol. 151, no. 1, pp. 153-166, 2012.

[7] F. Sallusto, J. Geginat, and A. Lanzavecchia, "Central memory and effector memory $\mathrm{T}$ cell subsets: function, generation, and maintenance," Annual Review of Immunology, vol. 22, pp. 745763, 2004.

[8] M. Miyara, Y. Yoshioka, A. Kitoh et al., "Functional delineation and differentiation dynamics of human CD4+ T cells expressing the FoxP3 transcription factor," Immunity, vol. 30, no. 6, pp. 899-911, 2009.

[9] H. Fujii, A. Arakawa, A. Kitoh et al., "Perturbations of both nonregulatory and regulatory FOXP3 + T cells in patients with malignant melanoma," British Journal of Dermatology, vol. 164, no. 5, pp. 1052-1060, 2011.

[10] S. Sakaguchi, M. Miyara, C. M. Costantino, and D. A. Hafler, "FOXP $3^{+}$regulatory T cells in the human immune system," Nature Reviews Immunology, vol. 10, no. 7, pp. 490-500, 2010.

[11] M. Miyara, G. Gorochov, M. Ehrenstein, L. Musset, S. Sakaguchi, and Z. Amoura, "Human FoxP3+ regulatory T cells in systemic autoimmune diseases," Autoimmunity Reviews, vol. 10, no. 12, pp. 744-755, 2011.

[12] W.-Z. Wei, G. P. Morris, and Y.-C. M. Kong, "Anti-tumor immunity and autoimmunity: a balancing act of regulatory $\mathrm{T}$ cells,' Cancer Immunology, Immunotherapy, vol. 53, no. 2, pp. 73-78, 2004

[13] O. Saitoh and Y. Nagayama, "Regulation of Graves' hyperthyroidism with naturally occurring $\mathrm{CD} 4+\mathrm{CD} 25+$ regulatory $\mathrm{T}$ cells in a mouse model," Endocrinology, vol. 147, no. 5, pp. 24172422, 2006.

[14] P. Verginis, H. S. Li, and G. Carayanniotis, "Tolerogenic semimature dendritic cells suppress experimental autoimmune thyroiditis by activation of thyroglobulin-specific $\mathrm{CD} 4^{+} \mathrm{CD} 25^{+}$ T cells," The Journal of Immunology, vol. 174, no. 11, pp. 74337439, 2005.

[15] C. Li, J. Yuan, Y.-F. Zhu et al., "Imbalance of Th17/treg in different subtypes of autoimmune thyroid diseases," Cellular Physiology and Biochemistry, vol. 40, no. 1-2, pp. 245-252, 2016.

[16] H. Xue, X. Yu, L. Ma et al., "The possible role of $\mathrm{CD} 4^{+} \mathrm{CD} 25$ (high)Foxp $3^{+} / \mathrm{CD} 4^{+} \mathrm{IL}-17 \mathrm{~A}^{+}$cell imbalance in the autoimmunity of patients with Hashimoto thyroiditis," Endocrine Journal, vol. 50, no. 3, pp. 665-673, 2015.

[17] A. Rodríguez-Muñoz, M. Vitales-Noyola, A. Ramos-Levi, A. Serrano-Somavilla, R. González-Amaro, and M. Marazuela, "Levels of regulatory $\mathrm{T}$ cells CD69+NKG2D+IL-10+ are increased in patients with autoimmune thyroid disorders," Endocrine Journal, vol. 51, no. 3, pp. 478-489, 2016.

[18] P. Pandiyan, L. Zheng, S. Ishihara, J. Reed, and M. J. Lenardo, "CD $4{ }^{+} \mathrm{CD} 25^{+}$Foxp $^{+}$regulatory $\mathrm{T}$ cells induce cytokine deprivation-mediated apoptosis of effector $\mathrm{CD}^{+} \mathrm{T}$ cells," Nature Immunology, vol. 8, no. 12, pp. 1353-1362, 2007.

[19] C. L. Maynard, L. E. Harrington, K. M. Janowski et al., "Regulatory $\mathrm{T}$ cells expressing interleukin 10 develop from Foxp3+ and Foxp3- precursor cells in the absence of interleukin 10," Nature Immunology, vol. 8, no. 9, pp. 931-941, 2007.

[20] L. W. Collison, C. J. Workman, T. T. Kuo et al., "The inhibitory cytokine IL-35 contributes to regulatory T-cell function," Nature, vol. 450, no. 7169, pp. 566-569, 2007.

[21] D. Getnet, J. F. Grosso, M. V. Goldberg et al., "A role for the transcription factor Helios in human CD4+CD25+ regulatory T cells," Molecular Immunology, vol. 47, no. 7-8, pp. 1595-1600, 2010.

[22] H. Takatori, H. Kawashima, A. Matsuki et al., "Helios enhances treg cell function in cooperation with FoxP3," Arthritis \& Rheumatology, vol. 67, no. 6, pp. 1491-1502, 2015.

[23] R. A. Gottschalk, E. Corse, and J. P. Allison, "Expression of Helios in peripherally induced Foxp $3^{+}$regulatory T cells," The Journal of Immunology, vol. 188, no. 3, pp. 976-980, 2012.

[24] S. Dai, R. Jia, X. Zhang, Q. Fang, and L. Huang, "The PD-1/PDLs pathway and autoimmune diseases," Cellular Immunology, vol. 290, no. 1, pp. 72-79, 2014.

[25] M. Marazuela, M. A. García-López, N. Figueroa-Vega et al., "Regulatory T cells in human autoimmune thyroid disease," The Journal of Clinical Endocrinology \& Metabolism, vol. 91, no. 9, pp. 3639-3646, 2006.

[26] A. Nakano, M. Watanabe, T. Iida et al., "Apoptosis-induced decrease of intrathyroidal $\mathrm{CD} 4{ }^{+} \mathrm{CD} 25^{+}$regulatory $\mathrm{T}$ cells in autoimmune thyroid diseases," Thyroid, vol. 17, no. 1, pp. 25-31, 2007.

[27] C. Mao, S. Wang, Y. Xiao et al., "Impairment of regulatory capacity of $\mathrm{CD} 4+\mathrm{CD} 25+$ regulatory $\mathrm{T}$ cells mediated by dendritic cell polarization and hyperthyroidism in Graves' disease," The Journal of Immunology, vol. 186, no. 8, pp. 4734-4743, 2011.

[28] K. He, Y. Hu, and X. Mao, "Abnormal proportions of immune regulatory cells and their subsets in peripheral blood of patients 
with Graves' disease," Chinese Journal of Cellular and Molecular Immunology, vol. 30, no. 11, pp. 1190-1193, 2014.

[29] M. Klatka, L. Kaszubowska, E. Grywalska et al., "Treatment of Graves' disease with methimazole in children alters the proliferation of Treg cells and CD3+ T lymphocytes," Folia Histochemica et Cytobiologica, vol. 52, no. 1, pp. 69-77, 2014.

[30] M. Klatka, E. Grywalska, M. Partyka, M. Charytanowicz, E. Kiszczak-Bochynska, and J. Rolinski, "Th17 and Treg cells in adolescents with Graves' disease. Impact of treatment with methimazole on these cell subsets," Autoimmunity, vol. 47, no. 3, pp. 201-211, 2014.

[31] K. Matsuzawa, S. Izawa, T. Okura et al., "Implications of FoxP3-positive and-negative CD4+ CD25+ T cells in Graves' ophthalmopathy," Endocrine Journal, vol. 63, no. 8, pp. 755-764, 2016.

[32] P. Pawlowski, K. Grubczak, J. Kostecki et al., "Decreased frequencies of peripheral blood CD4+CD25+CD127-Foxp3+ in patients with graves' disease and graves' orbitopathy: enhancing effect of insulin growth factor-1 on treg cells," Hormone and Metabolic Research, vol. 49, no. 3, pp. 185-191, 2017.

[33] Y. Liu, X. Tang, J. Tian et al., "Th17/Treg cells imbalance and GITRL profile in patients with hashimoto's thyroiditis," International Journal of Molecular Sciences, vol. 15, no. 12, pp. 21674-21686, 2014.

[34] J.-R. Kim, J.-N. Chae, S.-H. Kim, and J.-S. Ha, "Subpopulations of regulatory $\mathrm{T}$ cells in rheumatoid arthritis, systemic lupus erythematosus, and behcet's disease," Journal of Korean Medical Science, vol. 27, no. 9, pp. 1009-1013, 2012.

[35] A. Mathian, C. Parizot, K. Dorgham et al., "Activated and resting regulatory $\mathrm{T}$ cell exhaustion concurs with high levels of interleukin-22 expression in systemic sclerosis lesions," Annals of the Rheumatic Diseases, vol. 71, no. 7, pp. 1227-1234, 2012.

[36] A. S. S. Khaja, S. M. Toor, H. E. Salhat, B. R. Ali, and E. Elkord, "Intratumoral FoxP3+Helios+ regulatory T Cells upregulating immunosuppressive molecules are expanded in human colorectal cancer," Frontiers in Immunology, vol. 8, p. 619, 2017.

[37] T. Alexander, A. Sattler, L. Templin et al., "Foxp3 ${ }^{+}$Helios $^{+}$ regulatory $\mathrm{T}$ cells are expanded in active systemic lupus erythematosus," Annals of the Rheumatic Diseases, vol. 72, no. 9, pp. 1549-1558, 2013.

[38] A. H. Sharpe, E. J. Wherry, R. Ahmed, and G. J. Freeman, "The function of programmed cell death 1 and its ligands in regulating autoimmunity and infection," Nature Immunology, vol. 8, no. 3, pp. 239-245, 2007.

[39] W. Wang, R. Lau, D. Yu, W. Zhu, A. Korman, and J. Weber, "PD1 blockade reverses the suppression of melanoma antigenspecific CTL by CD $4^{+} \mathrm{CD} 25^{\mathrm{Hi}}$ regulatory T cells," International Immunology, vol. 21, no. 9, pp. 1065-1077, 2009.

[40] L. M. Francisco, P. T. Sage, and A. H. Sharpe, "The PD-1 pathway in tolerance and autoimmunity," Immunological Reviews, vol. 236, no. 1, pp. 219-242, 2010.

[41] S. Amarnath, C. W. Mangus, J. C. M. Wang et al., "The PDL1-PD1 axis converts human TH1 cells into regulatory T cells," Science Translational Medicine, vol. 3, no. 111, Article ID 111ra120, 2011.

[42] A. H. Sharpe and K. E. Pauken, "The diverse functions of the PD1 inhibitory pathway," Nature Reviews Immunology, vol. 18, no. 3, pp. 153-167, 2018.

[43] D. Franceschini, M. Paroli, V. Francavilla et al., "PD-L1 negatively regulates CD4+CD25+Foxp3+ Tregs by limiting STAT5 phosphorylation in patients chronically infected with $\mathrm{HCV}$,"
The Journal of Clinical Investigation, vol. 119, no. 3, pp. 551-564, 2009.

[44] H. Kristjansdottir, K. Steinsson, I. Gunnarsson, G. Gröndal, K. Erlendsson, and M. E. Alarcón-Riquelme, "Lower expression levels of the programmed death 1 receptor on CD4+CD25+ T cells and correlation with the PD-1.3A genotype in patients with systemic lupus erythematosus," Arthritis \& Rheumatology, vol. 62, no. 6, pp. 1702-1711, 2010.

[45] M. K. Tembhre, A. S. Parihar, V. K. Sharma, A. Sharma, P. Chattopadhyay, and S. Gupta, "Alteration in regulatory T cells and programmed cell death 1-expressing regulatory $\mathrm{T}$ cells in active generalized vitiligo and their clinical correlation," British Journal of Dermatology, vol. 172, no. 4, pp. 940-950, 2015.

[46] H. T. Jin, R. Ahmed, and T. Okazaki, "Role of PD-1 in regulating T-cell immunity," Negative Co-Receptors and Ligands, vol. 350, pp. 17-37, 2011. 


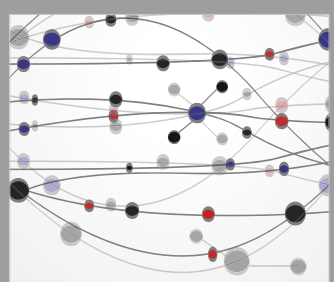

The Scientific World Journal
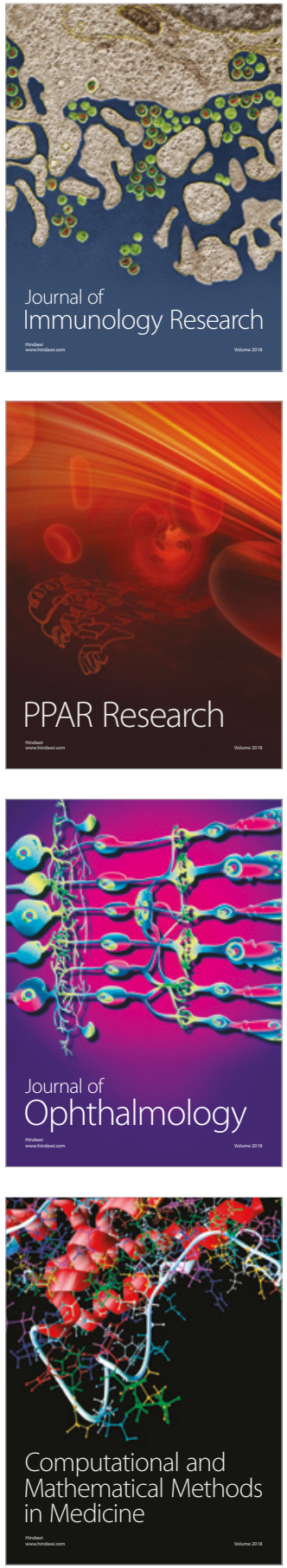

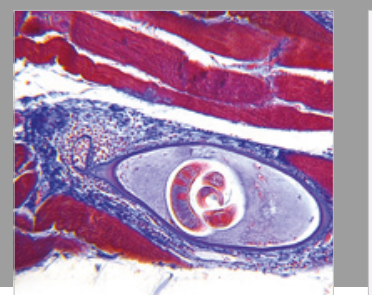

Gastroenterology Research and Practice

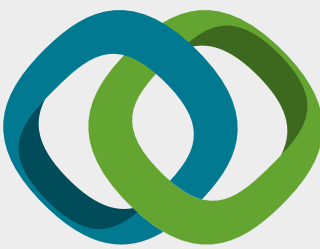

\section{Hindawi}

Submit your manuscripts at

www.hindawi.com
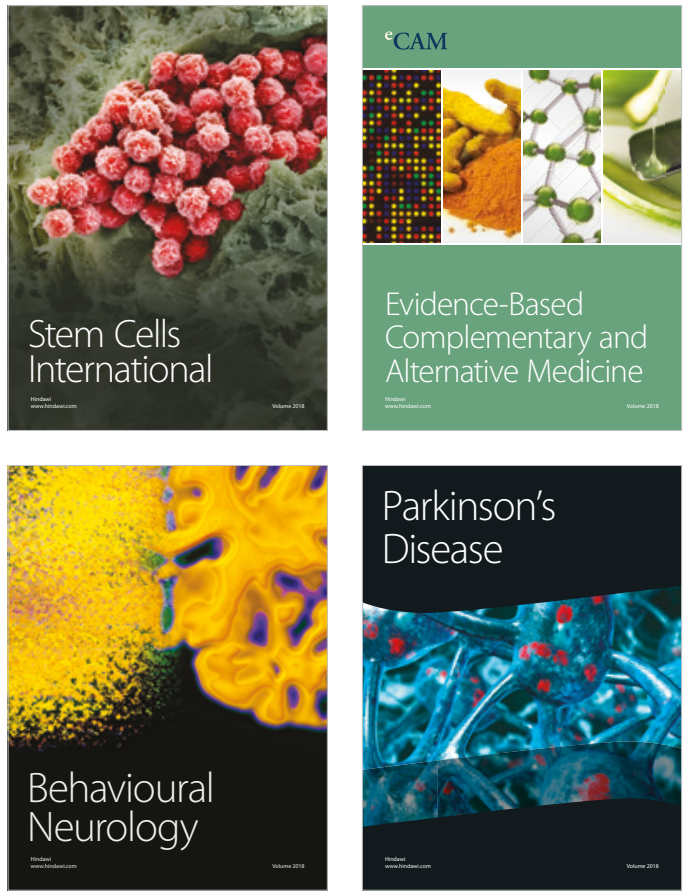

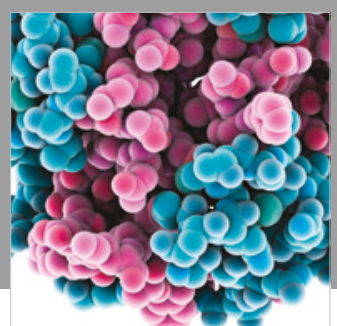

ournal of

Diabetes Research

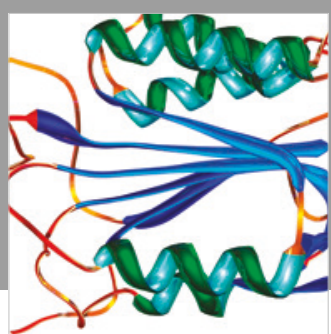

Disease Markers
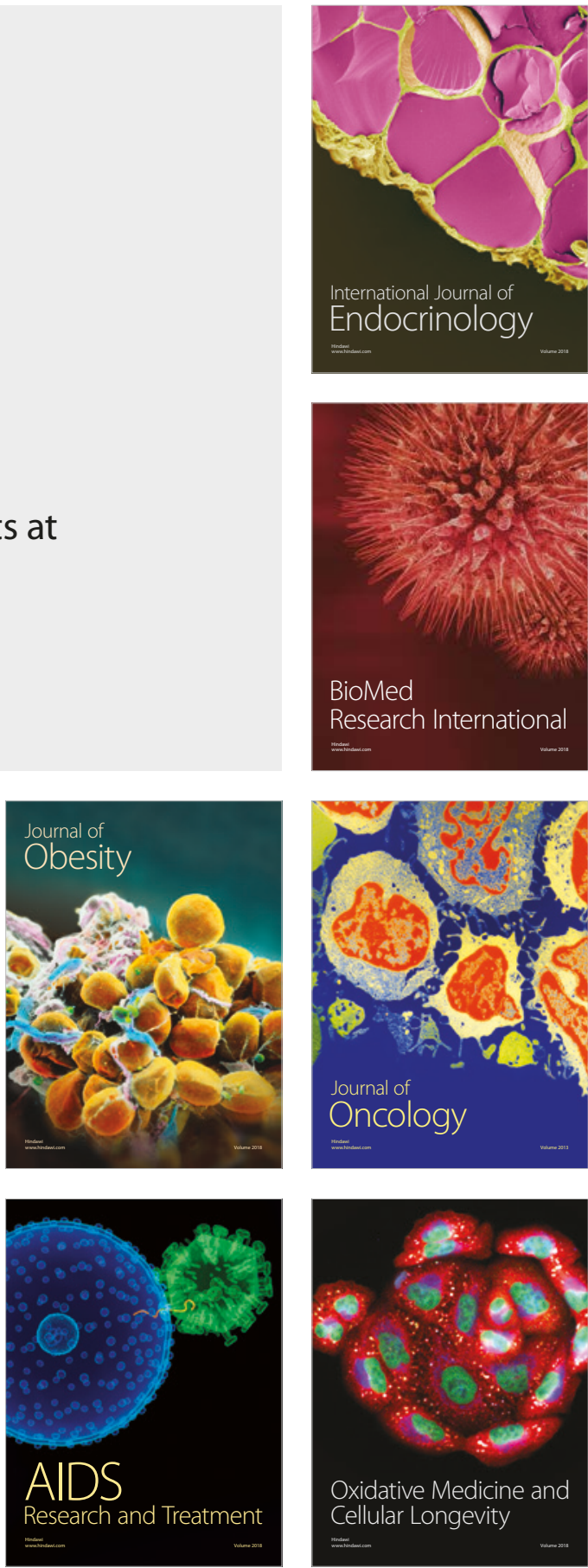